\title{
Research on mobile learning system construction based on smart phones
}

\author{
Nie Huiyu \\ Jilin baicheng normal college media institute \\ postcode 137000
}

Keywords: smart phones; mobile learning; system structure; cross-platform

\begin{abstract}
With the development of technology such as cloud computing, wireless networks and mobile devices, the application of mobile learning in the education of all types and at all levels is increasing popular. The importance will increasingly highlight: it is expected to become the primary entrance for the learners to digital learning world. In the face of the status quo of the smart phone with diversified operating system and differential characteristics, mobile learning system based on smart phone must solve cross-platform difficulties. From the points of view of cross-compilation, mobile web app, mixed mobile web app, mobile widgets, this article discusses the cross-platform key technologies of mobile learning system, summarizes the characteristics, the advantages and disadvantages of different technology, designs mobile learning system structure meeting the needs of different functions under different technical support, and discusses the construction plan of the cross-platform mobile learning system based on smart phone.
\end{abstract}

\section{Mobile learning theory}

Mobile learning is a new type of learning style born with development background of modern society, education, culture, science and technology. From the perspective of the organic combination of education and technology, mobile learning is defined as: learning with support of which covers all mobile devices, such as mobile phones, portable computers and other digital media devices; and learning in the society where a individual and knowledge have mobility ${ }^{[1]}$.

1) Mobile learning is the product of combination of digital learning and mobile computing, and it supports access to resources for learning activities anytime or anywhere. 2) Mobile learning is a learning way for learners to use mobile devices to learn in geographical spatial mobility and flexibility of time. 3) Mobile learning can be made in any location under the support of mobile devices, and mobile learning devices must have the ability to effectively present learning resources and two-way communication. 4) Mobile learning is the combining site of digital learning and mobile computing, and it can provide learning experience anytime or anywhere. 5) Mobile learning is now based on the mature wireless communication network and wireless multimedia, and learners realize the interactive teaching through mobile terminal. 6) Mobile learning makes learners use mobile portable devices to conduct learning activities without the time and place limitations.

According to these ideas, mobile learning has these characteristics: mobility (anytime or anywhere), based on the mobile network, interactivity, digital learning.

\section{Technology requirements of the mobile learning system based on smart phone}

On the basis of mobile development technology at the present stage, fully considering the characteristics of the mobile terminal, and running smoothly on the different mobile terminals, will create a learning environment for learners of different mobile terminals anytime and anywhere, and they can conduct autonomous learning online or offline, getting seamless learning experience.

The operating system of smart phone is diversified, such as Android, iOS, WP 7, BlackBerry, WebOS, etc; The difference of phone own features, such as screen size, input method, displaying ability is huge. Solution to the problem is to realize the relationship between resources, interaction, application function and learning itself, and try to extract open, reusable format and standards, design cross-platform mobile learning system based on smart phones, and achieve relatively 
accordance between different learners and platforms. Cross-platform concept is an important concept in software development, which is not dependent on the operating system, but don't trust hardware environment. The application of development in an operating system can still run in another operating system.

The development ways of cross-platform Mobile learning system based on smart phone mainly have Cross-compilation Compilation, Mobile Web App, Hybrid Mobile Web Apps and Mobile Widgets ${ }^{[2]}$.Different methods have different characteristics, adapted to the mobile learning system with different functions.

\section{Cross-compiled mobile learning system}

3.1 Cross-compilation technology (a coding forms the local app) cross-compilation will separate compiled environment (host platform) and the target environment platform (purpose platform), which is make use of cross compile chain to generate executable file on the host platform to form this app. Developers can use third party language, like JavaScript, Ruby or Java, with the help of a special kind of Application program interface (API) to build the application program, and to design the user interface, data presentation and business logic, which can run on different mobile terminal platform.

The technology advantages are: performance is good, and the program can run locally on the mobile terminal; User experience is good, and the feeling is like running a common application; The formative app integrates the mobile phones build-in function such as camera, memory card, etc. The biggest drawback is complex, because of the difficulty of a cross-compiler construction, choosing software versions consistent with the mobile platform and operating system and compile switch option is tedious.

Improved cross-compilation is a virtual machine (VM) technology. Virtual machine can abstracts the details of the target platform from the application, achieve completed hardware system functions by software simulation, and establish the interoperability between operating systems and applications. Virtual machine inherits some advantages of the cross compilation, and the biggest advantage is portability: when a device adds new features and needs the application interface support, the virtual machine is more easy to maintain and more flexible to extend. But the operating efficiency application was slightly lower.

3.2 Cross-platform development tools of cross-compilation

Cross-platform development tool Titanium adopted cross compilation techniques. It uses JavaScript write the code base to compile native code and package according to different target platforms. Support platform: iOS, Android, Black Berry. Advantage lies in quick local code output; Easy to install and start; Intuitive application management environment; Free of charge. The disadvantage is that supported mobile operating system are not widely; You must install the software development kit; No the clouds compilation; Additional support and training are for a fee.

Cross-platform development tool Rhomobile adopted cross-compilation technology based on the virtual machine, which run a single source base written by Ruby interpreter run Ruby and HTML/JavaScript/CSS on the terminal Ruby interpreter. Rhode running time (including interpreter) is determined by the local app, and provides the cross-platform general ability to abstractly access device. Rhomobile provides a complete set of tool for mobile application development, including RhoSync used for data synchronization between foreground and background, RhoHub used for the cloud compilation and RhoGallery management applications. Support platform have iOS, Android, BlackBerry, Symbian, Windows Mobile 6.5. Advantage lies in open source, model-view-controller architecture, the cloud compilation, products for free. The disadvantage is to need completely rebuilding to update HTML/JavaScript code, and need to learn a Ruby, not produce only local source code package and limited application process.

3.3 Architecture and function selection of cross-compiled mobile learning system

Mobile learning system based on smart phone choose Titanium or Rhomobile tool, and use the $\mathrm{C} / \mathrm{S}$ (client/server) structure to design mobile learning system ${ }^{[3]}$, as shown in figure 1.Smart phone installation used client program of cross-compiled technology. When running, the client established 
the connection with server by means of wireless internet to make corresponding data communication. Mobile learning resources (hypertext, images, streaming media) are distributed by the server, which implement the maintenance and update of the data in the database. System development has a certain difficulty.

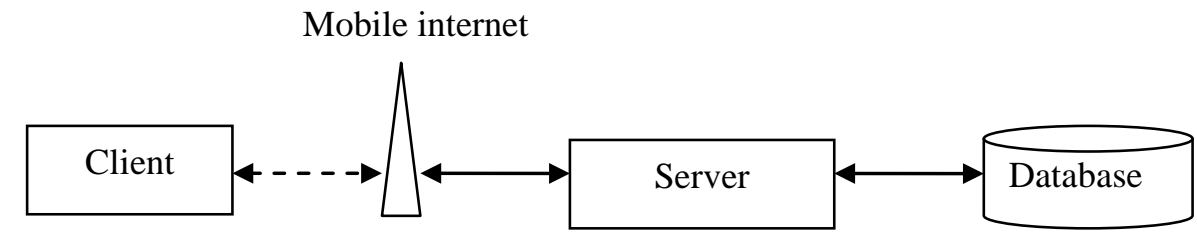

Figure 1 Architecture of mobile learning system on cross-compilation technologies

Cross-platform mobile learning system supported by cross-compiled technical has powerful functions, real life situation and positive interaction, suitable for mobile learning of all basic functions, especially suitable for the mobile learning with rich image, augmented reality, exploring, game and study function.

\section{Mobile learning system of mobile web app (running in a mobile browser)}

4.1 Mobile web app technology

Running mobile web app in the smart phone mobile browsers is a common cross-platform technology method. Developers using standard Web technologies, such as HTML, CSS and JavaScript to build the application. As a result of advanced features of HTML 5 and CSS 3, including embedded SQL database, local storage, animation, network socket and video playing, mobile Web application is more and more like a native app on the appearances and behaviors.

Web app typically run in a separate mobile Web browser (pure network).Advantages lie in: simple deployment, rapid development, easy to use, and directly using built-in browser of mobile phone to input URL to establish an effective data connection. The disadvantages are: on the one hand, the user experience is poorer, lack of interaction; On the other hand, it can't make use of cell phones built-in function.

4.2 Architecture and function selection of mobile learning system of mobile web app

Mobile learning system based on smart phones uses the mobile web app technology, and adopts $\mathrm{B} / \mathrm{S}$ architecture. Developers use standard web development language to publish to the Internet, then teachers and learners can access the mobile platform to carry out the teaching and learning directly through the browser, and don't need to install any software, and various functional module designs are easy to use, to get started, as shown in figure 2.

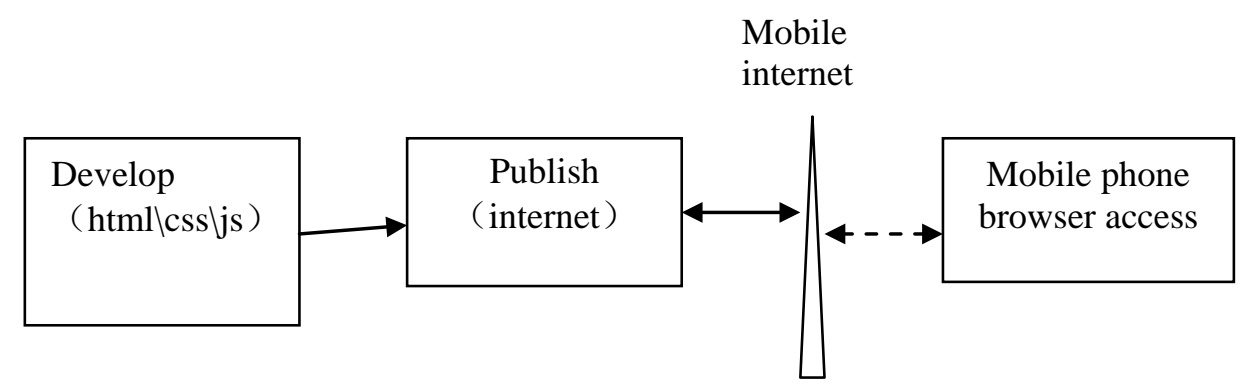

Figure 2 Development process of mobile web application

System development is simple, convenient and fast. Due to the limitations of its own, mobile web app is suitable for mobile learning with knowledge transmission as the main function, and is not suitable for applications with frequent interaction, intensive CPU and rich vision, such as games, augmented reality and video conference. 


\section{Mobile learning system on mixed mobile web app}

5.1 Mixed mobile web app

Mixed mobile web application is to embed the browser into the local app. In the hybrid model, the native program of thin body type built bridges between the terminal operating systems and services, and the web apps operated in its internal. Web apps installed on the local cache, no longer need effective data connection, improving the speed and the reaction ability. The communications between web applications and local application can be realized by custom API JavaScript. The technology will have the advantages of good user interaction experience of local application and cross-platform development of web application, which can not only provide broad support for different terminal equipment, and without reconstruction resource content. In addition, it also allows developers to make up for the inadequacy of the mobile browser on the phone by adding additional device built-in function. Poor user experience will be a great disadvantage, because web browser only uses standard network elements, impossible to simulate the local user interface, and using of mobile terminal function (such as contacts, memory card and sensor) is usually limited.

5.2 Cross-platform development tools of mixed mobile web app

Cross-platform development tool PhoneGap and AppMobi apply technology architecture of hybrid mobile web applications. The PhoneGap application is built by HTML 5 and CSS and JavaScript, which customizes scripts or IDE compilation by mobile operating system, carries out in the browser of thin body type app bag.

Independent JavaScript API can access to mobile terminal built-in function, and supports almost all popular mobile operating systems, such as iOS, Android, BlackBerry, Symbian, etc. Advantage lies in open source, providing local source code package, easy to customize; Providing a simple embedded base, easy to develop; A wide range of platform support; app written in HTML, JavaScript, and CSS is easy to develop; The cloud compilation (beta version).Drawback is that you must install/use separate integrated development environment (IDE) and software development kit, different operating systems have different installation, it lacks of support for local UI components, design patterns and peripheral tools. And AppMobi introduced a new development tool XDK, a application allowing developers to use HTML5 to build the Internet and mobile platform, supporting iOS and Android. Advantage is free XDK and the clouds compilation of the initial application. The disadvantage is to only support limited mobile operating system; The application would update fee during the later period; Requiring a Google browser and Java6 platform.

5.3 System architecture and function section of mobile learning system for mixed mobile web app

Because mixed mobile web app combines the advantage of the local app and mobile web app, basically suitable for all the features of mobile learning system. It is preferred technique to develop mobile learning, and is the development trend in the future. Developers use HTML, CSS, JavaScript, standard web language to develop, and compile into system program adapting to the target terminal under the different compiled environment, as shown in figure 3. In the system architecture, the local app embedded in the webapp, both suit for knowledge transmission, human-computer interaction, also suitable for situation display, as shown in figure 4 . 


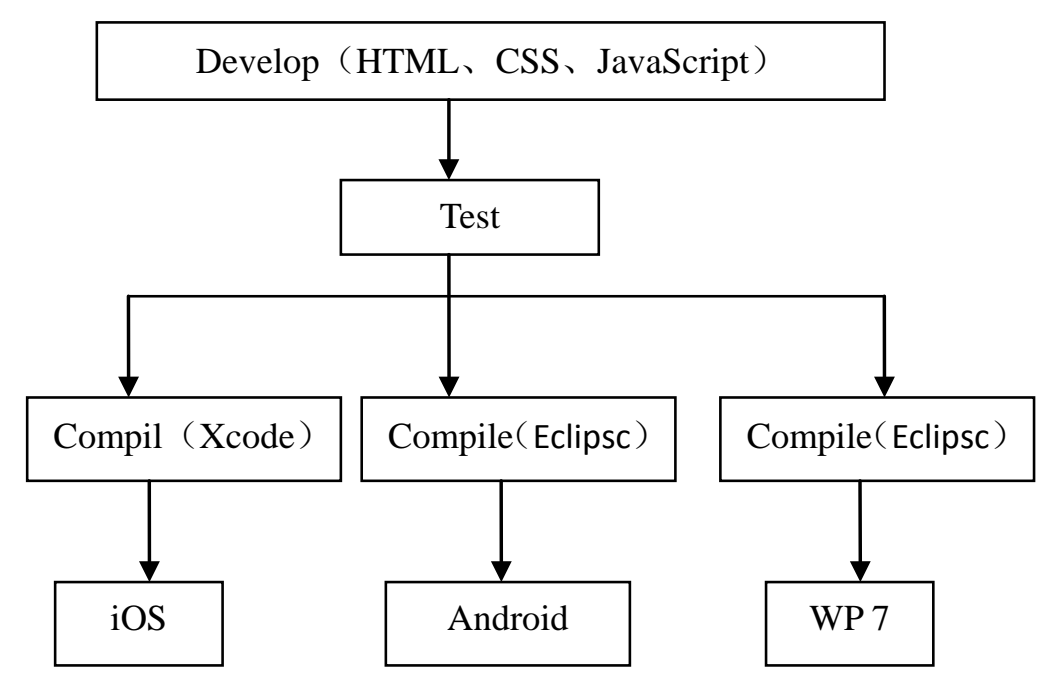

Figure 3 Development process of mixed mobile web app

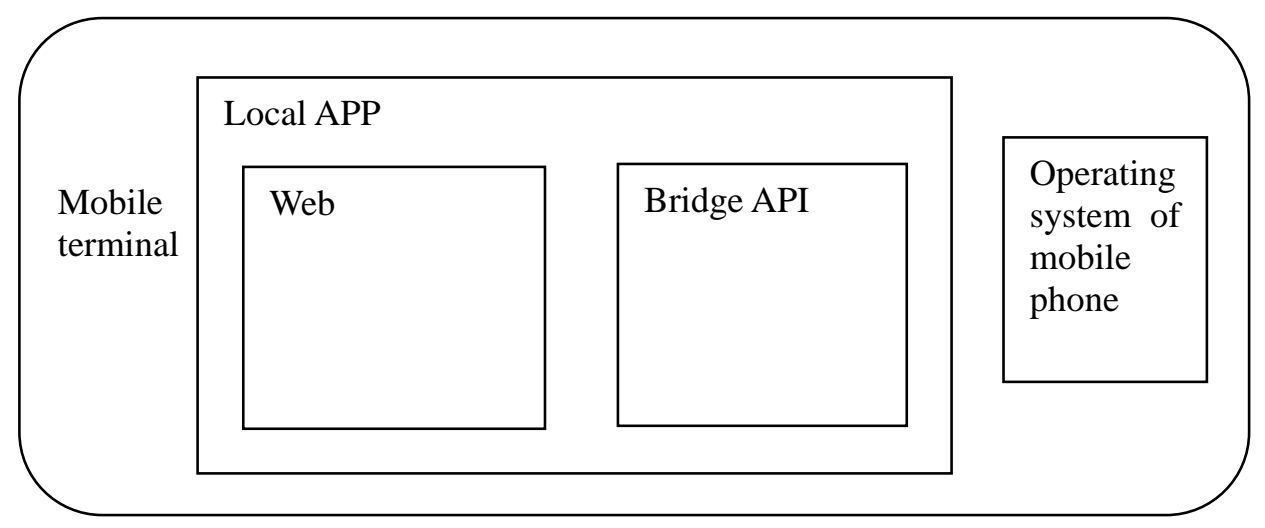

Figure 4 Framework of mixed mobile web app

\section{Mobile learning system for mobile widgets}

\subsection{Mobile widgets}

Mobile Widget is a kind of interactive widgets, providing users with a single personalized services, such as the latest news, weather, calendar, maps, dictionaries, calculators, etc., often appearing on the major screen or virtual desktop of the phone. It faces end users with a lightweight, intuitive way ${ }^{[4]}$. Widget is a standard and small app developed by HTML, JavaScript, and CSS technology. Advantage is more convenient development; Volume is smaller; User experience is flexible; Cross-platform. JavaScript API usually provided by the platform makes access the function of devices, such as cameras, contacts, and storage, like a typical native applications, and this is very similar with mixed mobile web app, then the only difference is the encapsulation of terminal function and access ways, and package and access method of Widget API are much more than the cross-platform library customized based on HTML $5^{[5]}$.

6.2 Architecture and function section of mobile learning system for mobile widgets

The flexibility of mobile widgets matches with learning tool feature of mobile learning system, therefore it is very suitable for developing learning small tools, such as professional glossary, thesaurus, etc. When developing, developers can use HTML, JavaScript, CSS, and XML language, and the system structure is shown in figure 5 . 


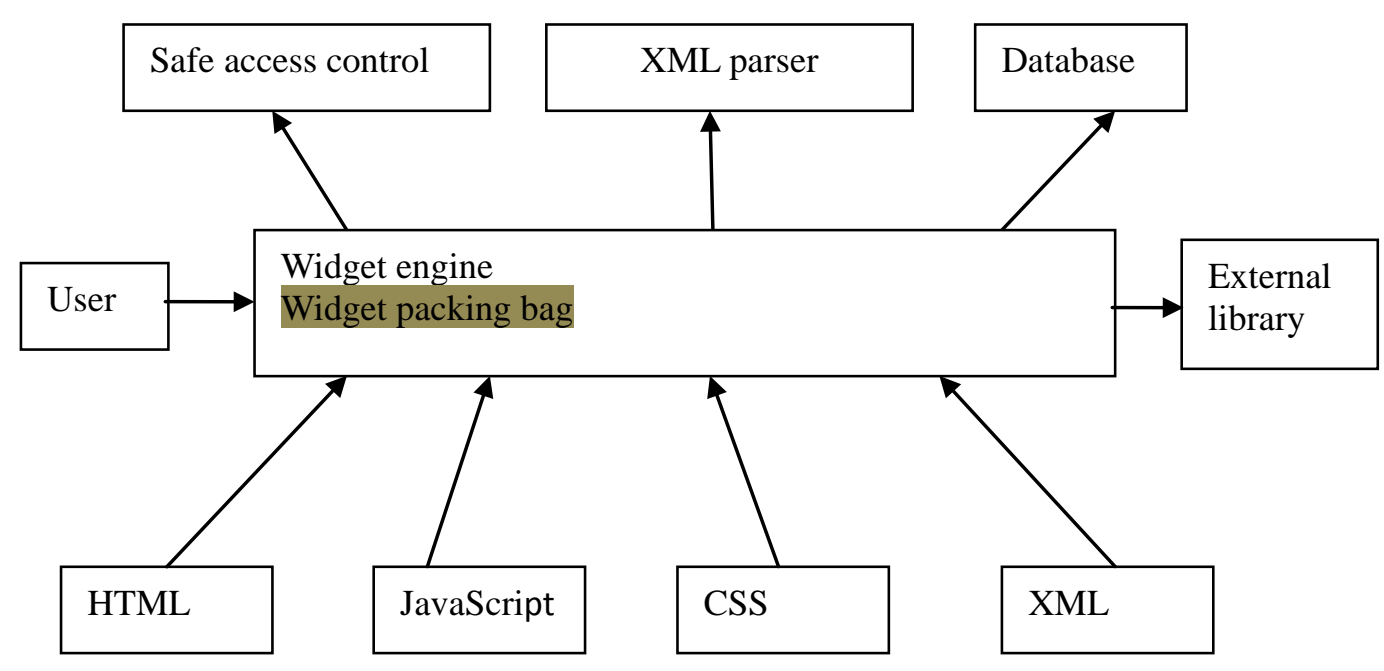

Figure 5 Mobile learning system structure drawing of mobile widgets

(1) Safe access control: using authentication, authorization and access control mechanism to improve safety.

(2) XML parser: parsing the XML file when handling data structure.

(3) Database: storing and updating the data.

(4) External library: using mature tested external libraries to improve the function.

\section{Conclusion}

According to the function of the mobile learning system, cross-platform technologies such as cross-compilation, mobile webapp, mixed mobile web app, mobile widgets can be applied to different mobile learning system design and development, of course, they can be also combined, offering more abundant development kit. JavaScript is a kind of commonly used languages of developing system, needing to choose Mobile JavaScript framework, and the more popular are jQTouch, jQuery Mobile and Sencha Touch. Aiming at the new type of computing environment with rapid development for the future, mobile learning will play a more and more important role, and in the new environment mobile learning will have a better and more complicated application mode. All in all, according to the technology development and learning needs, there is still a long way to grope for constantly developing new function of mobile learning and choosing suitable new technology.

\section{References}

[1] Fu Jian, Yang Xue. Bird's-eye view on theory research and practice emerged of domestic mobile learning in recent years [J]. China Audio-visual Education, 2009, (7)

[2] Liu Gang, Zhu Peijun, Li Huiyun, etc. Research on intelligent terminal cross-platform application and development technology [J]. Journal of Telecom Science, 2012 (5)

[3] Xie Panke. Design and implementation of mobile learning system based on Android [D]. Wuhan: Central China Normal University, 2011.

[4] Guo Wenli. Mobile widgets and its application research progress [J]. Journal of Library Journal, 2012, 31 (1)

[5] Zhang Kaiyong. architecture of new application model of mobile library phone information service - based on the analysis of the mobile widgets [J]. Journal of Library Science Research, 2012 (20) 